\title{
Diagnostic Accuracy of Serum and Urine S100A8/A9 and Serum Amyloid A in Probable Acute Abdominal Pain at Emergency Department
}

\author{
Arash Forouzan (D), ${ }^{1}$ Kambiz Masoumi $(\mathbb{D}),{ }^{1}$ Fakher Rahim $\left(\mathbb{D},{ }^{2,3}\right.$ Meisam Moezzi, ${ }^{1}$ \\ Ali Khavanin, ${ }^{1}$ Nastaran Ranjbari, ${ }^{4}$ Malehi Amal Saki, ${ }^{5}$ Amirali Fallah Amoli, ${ }^{1}$ \\ Niloofar Akhiani, ${ }^{1}$ and Fatemeh Ghourchian ${ }^{1}$ \\ ${ }^{1}$ Department of Emergency Medicine, Imam Khomeini General Hospital, Ahvaz Jundishapur University of Medical Sciences, \\ Ahvaz, Iran \\ ${ }^{2}$ Health Research Institute, Research Center of Thalassemia \& Hemoglobinopathy, Ahvaz Jundishapur University of Medical Sciences, \\ Ahvaz, Iran \\ ${ }^{3}$ Metabolomics and Genomics Research Center, Endocrinology and Metabolism Molecular - Cellular Sciences Institute, \\ Tehran University of Medical Sciences, Tehran, Iran \\ ${ }^{4}$ Department of Pathology, Imam Khomeini General Hospital, Ahvaz Jundishapur University of Medical Sciences, Ahvaz, Iran \\ ${ }^{5}$ Department of Biostatics and Epidemiology, School of Public Health, Ahvaz Jundishapur University of Medical Sciences, Ahvaz, Iran
}

Correspondence should be addressed to Kambiz Masoumi; emdajums@yahoo.com

Received 15 December 2017; Revised 19 March 2018; Accepted 17 April 2018; Published 3 July 2018

Academic Editor: Shih-Ping Hsu

Copyright (C) 2018 Arash Forouzan et al. This is an open access article distributed under the Creative Commons Attribution License, which permits unrestricted use, distribution, and reproduction in any medium, provided the original work is properly cited.

Study Design. This study was performed to investigate the diagnostic values of some inflammatory biomarkers in abdominal pain. Methods. Patients over 18 years of age with acute recent abdominal pain who presented to the Emergency Department were evaluated. Serum and urinary samples were taken and evaluated for serum and urine S100A8/A9 and serum amyloid A. All patients were referred to a surgeon and were followed up until the final diagnosis. In the end, the final diagnosis was compared with the levels of biomarkers. Results. Of a total of 181 patients, 71 underwent surgery and 110 patients did not need surgery after they were clinically diagnosed. Mean levels of serum and urine S100A8/A9 had a significant difference between two groups, but serum amyloid A did not show. The diagnostic accuracy of serum S100A8/A9, urine S100A8/A9, and serum amyloid A was $86 \%, 79 \%$, and $50 \%$, respectively, in anticipation of the need or no need for surgery in acute abdominal pain. Conclusions. Our study showed that in acute abdominal pain, serum and urine S100A8/A9 can be useful indicators of the need for surgery, but serum amyloid A had a low and nonsignificant diagnostic accuracy.

\section{Introduction}

Abdominal pain is one of the most common complaints that patients present to the hospital, making up $10 \%$ of all cases referred to the emergency department (ED) [1, 2]. Many patients have nonspecific abdominal pains that do not aid in diagnosing a specific disease and represent mild and self-limiting conditions to life-threatening diseases $[3,4]$. Nontraumatic acute abdominal pain is a medical emergency that lasts up to 5 days and does not result from trauma [5].

Acute abdominal pain is the most common cause of emergent surgery and one of the most frequent causes for referral to the ED as well as for counseling and admission of nontraumatic patients [6]. The most prevalent causes of emergent abdominal pain that need intervention within 24 hours include acute appendicitis, acute diverticulitis, acute cholecystitis, and bowel obstruction. The most prevalent 
causes of nonemergent cases include nonspecific abdominal pain and gastrointestinal diseases $[1,4]$.

Abdominal pain has a vague quality, because of the convergence of the second neuron of organs and abdominal structures in the posterior column of the spine. However, physical examination may reveal different findings in the patients and the location and the severity of pain may change over time, which makes the diagnosis of the causative agent a challenge for the physician $[1,7]$.

On time diagnosis plays a key role in deciding the treatment process of the patients, while delayed intervention leads to more complications, such as peritonitis and perforation, associated with higher rates of morbidity and mortality $[3,4,8,9]$.

Finding the causes of abdominal pain can be facilitated by imaging tools and methods. Ultrasonography, with medium sensitivity and specificity, can be used to diagnose the underlying disease, but it requires experienced and efficient operators, who may not always be available at health care facilities [10]. In the present times, the increasing use of computed tomography (CT) scans is often not required as the patient rarely gains any advantage and is exposed to harmful radiation and contrast nephropathy and has to bear additional costs $[7,11]$. However, despite the use of imaging methods, the diagnostic dilemma still remains challenging as it includes wrong diagnosis or unnecessary surgery $[4,5]$.

The use of new inflammatory biomarkers is a noninvasive and harmless method [12]. Some biomarkers can help find a proper diagnosis and reduce the use of imaging techniques, if they can be measured in serum, plasma, urine, or feces of the patients, quickly, and are accompanied by high sensitivity, reliability, and low costs $[3,13,14]$. Laboratory, animal, and human studies have shown the feasibility of serum and urine S100A8/A9 and serum amyloid A in inflammatory conditions $[3,7,13,15-19]$.

S100A8/A9 is a complex of the proteins S100A8 and S100A9 in mammals $[20,21]$. It has a high tendency to calcium, zinc, and manganese [22-24]. S100A8/A9 was introduced in 1980, for the first time, as an antimicrobial protein that destroys zinc in mammals [20,21,25]. Now, it has been found that its antibacterial and antifungal properties are due to its ability to destroy manganese [25]. This protein includes up to $60 \%$ of soluble proteins in neutrophil cytosols and, to a lesser extent, in monocytes; it includes macrophages and squamous epithelial cells [21-23]. Mammals secrete this protein during the inflammatory response. The exact mechanism for its secretion is unknown [26].

Serum amyloid A, a protein of HDL, is associated with the apolipoprotein family in plasma. It is mainly produced in the liver [27]. Some actions of these proteins include transferring cholesterol into the liver, calling immune cells to the site of inflammation, and inducing enzymes that break the extracellular matrix down. Small inflammatory responses can also increase the production of serum amyloid A. Plasma levels of serum amyloid A increase with injuries, infections, and inflammatory diseases, such as rheumatoid arthritis and Kawasaki, as well as with viral and autoimmune diseases and malignancies. It is an acute-phase reactant, increasing over a few hours after the inflammatory stimulus, which may increase even more than the C-reactive protein (CRP) $[1,9,28,29]$.

Although clinical findings and conventional biomarkers play a major role in the diagnosis of acute appendicitis or lower abdominal pain etiology, several studies suggest that S100A8/A9 in serum and in the intestine, alone or in combination with other biomarkers, can be a useful accessory and tool for clinical findings [7-30]. In other studies, using stool S100A8/A9 has been approved as a noninvasive method to differentiate the inflammatory versus noninflammatory causes of gastrointestinal diseases [13, 31-36]. Furthermore, Muhammad et al. reported that the diagnostic accuracy of serum amyloid A biomarker for acute appendicitis is higher than that of CRP [19].

Despite the technological progress in accuracy, rapidity, and the ease of diagnosing methods for abdominal pain, many challenges still remain to be addressed. Thus, an accessory diagnostic biomarker can help in the early diagnosis of cases that require emergent surgery to reduce the rates of morbidity and mortality, reducing the length of stay in the ED and lowering health care costs. The aim of this study was to evaluate the serum and urine S100A8/A9 and serum amyloid A biomarkers in patients presenting with acute abdominal pain in the ED, as a diagnostic biomarker for emergent surgery.

\section{Methods}

2.1. Participants. In this prospective diagnostic test accuracy (DTA) study, patients with recent abdominal pain referred to the ED of Imam Khomeini General Hospital, from January 2016 to January 2017, were enrolled. This study was approved and supported by the Jundishapur University of Medical Sciences, Ahvaz, Iran. The ethical committee of the university confirmed this study with ethical codes IR.AJUMS. REC1394.82, IR.AJUMS.REC1394.88, and IR.AJUMS.REC 1394.87, and all patients signed a written informed consent prior to enrollment.

2.2. Inclusion Criteria. Patients over 18 years of age, presenting to the ED with recent abdominal pain (under 72 hours), with abdominal tenderness and rebound tenderness on examination are included in the study.

2.3. Exclusion Criteria. Patients who are pregnant with flank pain alone, altered mental status, evidence of malignancy, history of autoimmune disease, inflammatory bowel disease, history of abdominal trauma, and surgery over the past two weeks are excluded in the study.

2.4. Test Methods. After the admission of the patients, necessary information, including age, sex, location of the pain, onset time of the pain, associated symptoms, and early clinical diagnosis (after history taking and physical examination), was recorded. Paraclinical measures, including blood sampling for the required tests and appropriate imaging methods, were taken. In addition, for all the patients entering the study, blood samples and urine specimens were sent for the measurement of serum and urine levels of S100A8/A9 and the serum level of amyloid A. The blood samples were centrifuged in the laboratory to separate their serum, kept 
at a temperature of $-20^{\circ} \mathrm{C}$, along with urine specimens, until the estimated sample size and the ultimate measurements were assessed. All patients were referred to a general surgeon and were carefully checked on. The final diagnosis of the patients who underwent surgery was made based on the pathology report by the responsible surgeon. For patients not requiring surgery, a final probable clinical diagnosis was based on the history, physical examination, paraclinical measures, and the final opinion of the general surgeon and consultants in different specialties, according to scientific and academic standards. After the estimated sample size was reached, all of the samples were analyzed for the serum and urine levels of S100A8/A9 and the serum level of amyloid A under the supervision of a pathologist. The normal values of biomarkers were determined using the human commercial ELISA kit of S100A8/A9 and serum amyloid A (Shanghai Crystal Day Biotech Co.) (normal serum S100A8/A9 and urine S100A8/A9: $100 \mu \mathrm{g} / \mathrm{cc}$, normal serum amyloid A: $9.02 \mu \mathrm{g} / \mathrm{cc}$ ). Finally, the white blood cell (WBC) count and the levels of serum and urinary biomarkers, based on the final diagnosis, were measured and compared in the surgical and nonsurgical groups. In total, 246 participants, comprising two subgroups according to acute abdominal pain status, were selected. The groups were named and composed as follows: PSI+/- (PSI, pain in the surgical intervention group), comprising subjects in whom acute abdominal pain remitted postsurgically, and PNSI+/- (PNSI, pain in the nonsurgical intervention group), consisting of those subjects in whom acute abdominal pain remitted after nonsurgical intervention.

2.5. Statistical Analysis. The collected data was performed by descriptive statistics, such as mean, standard deviation, frequency and percentage, and analytical statistics, including the nonparametric Mann-Whitney $U$ test and the Pearson correlation coefficient. The ROC curve analysis was used to evaluate the accuracy of blood cells and the serum and urine biomarkers in the diagnosis of acute abdominal pain requiring surgery. SPSS 21 software was used for the analysis. The significance level was considered to be less than 0.05 .

\section{Results}

In this study, a total of 246 patients were presented to the ED with clinical findings of acute abdominal pain; of these, 66 patients were excluded and 181 patients were studied and finally analyzed. Baseline characteristics of both the study groups are given in Table 1.

Of these, 71 patients underwent surgery (surgical group), and clinical diagnoses without the need for surgery were considered for 110 patients (nonsurgical group) (Figure 1).

The results showed that the levels of biomarkers were not statistically different between the surgical and the nonsurgical groups, in terms of mean age and gender distribution $(P<0.05)$.

In the surgical group (71 patients), the major final diagnosis included acute appendicitis (57.7\%), acute cholecystitis (15.4\%), and bowel obstruction (7\%), leaving $19.9 \%$ with other kinds of diagnoses including complicated ovarian cyst $(n=2)$, volvulus $(n=2)$, stomach perforation $(n=3)$, ectopic pregnancy $(n=1)$, colon tubu-adenomatosis tumor $(n=1)$, mesenteric ischemia $(n=1)$, pancreas abscess $(n=1)$, complicated ovarian tumor $(n=1)$, and duodenal perforation $(n=2)$ (Table 1 of Supplementary Material).

Our findings in this group showed that the mean levels of serum in S100A8/A9 were higher than normal, in the final diagnosis, except in mesenteric ischemia. Moreover, the mean urinary levels of S100A8/A9 were higher than normal in the final diagnosis except in volvulus. The mean serum levels of amyloid A, in the final diagnosis, were higher than normal, except in intestinal volvulus and ectopic pregnancy. Finally, the mean WBC count was higher than normal, in all cases, except in bowel obstruction (Table 2).

In the nonsurgical group (110 patients), the major final clinical diagnosis included ovarian cysts (22.7\%), acute pancreatitis $(10.9 \%)$, dyspepsia $(8.1 \%)$, acute cholecystitis (5.4\%), renal colic because of stones $(5.4 \%)$, and other diagnoses $(21.2 \%)$ : ectopic pregnancy $(n=3)$, acute cholangitis $(n=1)$, surgery site abscess $(n=1)$, gastritis $(n=2)$, acute hepatitis $(n=1)$, urinary tract infection $(n=2)$, biliary colic $(n=4)$, incarcerated periumbilical hernia $(n=1)$, varicocele $(n=1)$, pelvic inflammatory disease $(n=1)$, and mittelschmerz $(n=1)$ (Table 2 of Supplementary Material), thus leaving $26.3 \%$ with nonspecific abdominal pain (NSAP).

Our findings in this group showed that the mean serum and urine levels of S100A8/A9 were higher than normal in the final clinical diagnosis of acute pancreatitis, ectopic pregnancy, acute cholangitis, surgery site abscess, gastritis, acute hepatitis, ileus, and inflammatory pelvic disease and were normal in other diagnoses.

In all diagnoses, except renal colic because of stone, the mean serum levels of amyloid A were higher than normal. Finally, the mean WBC counts were higher than normal in patients with a final diagnosis of ovarian cysts, acute cholecystitis, acute pancreatitis, ectopic pregnancy, dyspepsia, ileus, urinary tract infection, biliary colic, and incarcerated periumbilical hernia (Table 3 ).

The results showed that the mean levels of serum and urine of S100A8/A9 biomarkers and WBC count were significantly higher in the surgical group than in the nonsurgical group. The results of the nonparametric Mann-Whitney $U$ test showed a statistically significant difference in these biomarkers between the surgical and the nonsurgical groups.

Among common diagnoses between the two groups (ovarian cysts, acute cholecystitis, and ectopic pregnancies), the mean WBC count was significantly higher in complicated ovarian cysts in the surgical group than in the nonsurgical group $(P<0.05)$. Moreover, the mean level of serum and urine S100A8/A9 in acute cholecystitis was significantly higher in the surgical group than in the nonsurgical group $(P<0.05)$. However, there was no significant difference in the mean levels of biomarkers in ectopic pregnancy between the two groups $(P<0.05)$ (Table 4$)$.

In our study, the data in Table 5 show the relationship between the time of onset of pain, levels of biomarkers, and WBC counts in the surgical and the nonsurgical groups.

The nonsurgical group had a statistically significant direct correlation between the mean time of onset of pain until the 
TABLE 1: Comparing baseline characteristics between the two groups of the study participants.

\begin{tabular}{|c|c|c|c|}
\hline Characteristics & Surgical $(n=71)$ & Nonsurgical $(n=110)$ & $P$ values \\
\hline Age (years)* & $37.04 \pm 15.86$ & $34.81 \pm 14.4$ & 0.102 \\
\hline \multicolumn{4}{|l|}{ Gender** } \\
\hline Male & $31(43.7 \%)$ & $44(40 \%)$ & 0.965 \\
\hline Female & $25(40.3 \%)$ & $66(60 \%)$ & 0.0541 \\
\hline WBC count $\left(\times 10^{9} / \mathrm{L}\right)^{*}$ & $12.7 \pm 10.14$ & $9.82 \pm 3.68$ & 0.0445 \\
\hline Serum S100A8/A9* & $208.17 \pm 101.5$ & $95.95 \pm 84.3$ & 0.0238 \\
\hline Urine S100A8/A9* & $147.64 \pm 44.5$ & $81.22 \pm 64.4$ & 0.0315 \\
\hline Serum amyloid $\mathrm{A}^{*}$ & $21.37 \pm 20.38$ & $20.95 \pm 23.09$ & 0.996 \\
\hline \multicolumn{4}{|l|}{ Pain onset } \\
\hline Admission day & $20(28.2 \%)$ & $31(28.2 \%)$ & 1.00 \\
\hline$<5$ days before admission & $42(59.2 \%)$ & $63(57.3 \%)$ & 0.998 \\
\hline 5-10 days before admission & $8(11.2 \%)$ & $13(11.8 \%)$ & 1.00 \\
\hline$>10$ days before admission & $1(1.4 \%)$ & $3(2.7 \%)$ & 0.985 \\
\hline
\end{tabular}

${ }^{*}$ Mean \pm SD. ${ }^{* *}$ Number (\%)

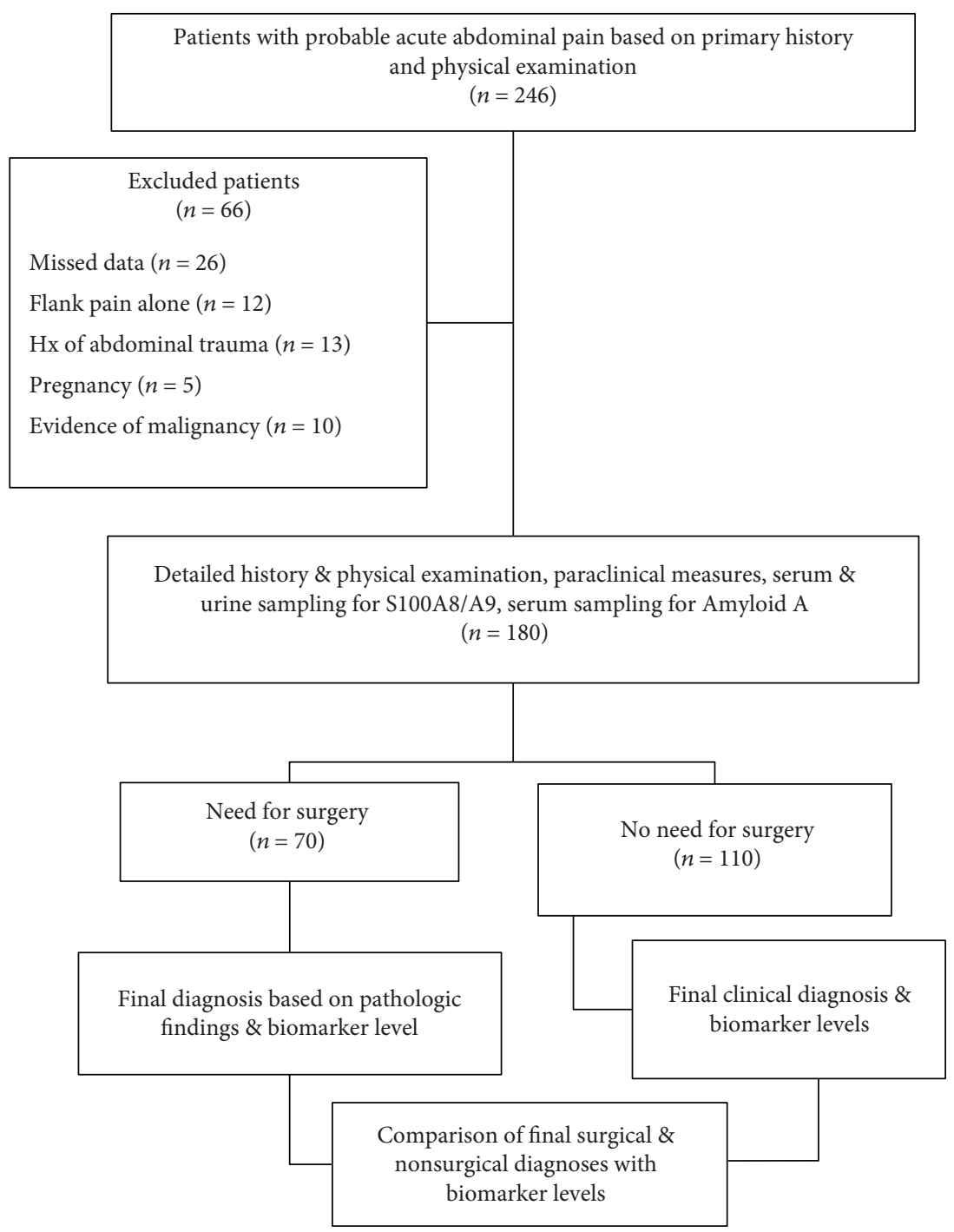

FIGURE 1: Schematic flowchart for the map of the study. 
TABLE 2: Distribution of mean white blood cell count and levels of biomarkers in different diagnoses based on pathological findings in the surgical group (in patients in which their numbers were $\geq 5$ ).

\begin{tabular}{|c|c|c|c|c|c|}
\hline Final diagnosis & Statistics & WBC count & Serum S100A8/A9 & Urine S100A8/A9 & Serum amyloid $\mathrm{A}$ \\
\hline \multirow{4}{*}{$\begin{array}{l}\text { Acute appendicitis } \\
N=41\end{array}$} & Mean & 11334.15 & 218.19 & 153.39 & 22.73 \\
\hline & Std. deviation & 3237.02 & 98.33 & 44.99 & 21.50 \\
\hline & Minimum & 3200.0 & 75.0 & 79.0 & 6.70 \\
\hline & Maximum & 20000.0 & 518.0 & 263.0 & 84.70 \\
\hline \multirow{4}{*}{$\begin{array}{l}\text { Acute cholecystitis } \\
N=11\end{array}$} & Mean & 19318.18 & 244.91 & 140.73 & 17.75 \\
\hline & Std. deviation & 24309.09 & 144.94 & 20.11 & 11.99 \\
\hline & Minimum & 10000.0 & 138.0 & 110.0 & 7.60 \\
\hline & Maximum & 92500.0 & 545.0 & 169.0 & 40.60 \\
\hline \multirow{4}{*}{$\begin{array}{l}\text { Gastrointestinal obstruction } \\
N=5\end{array}$} & Mean & 8980.0 & 173.80 & 121.20 & 9.62 \\
\hline & Std. deviation & 2534.17 & 61.63 & 45.53 & 3.09 \\
\hline & Minimum & 6200.0 & 92.0 & 47.00 & 5.50 \\
\hline & Maximum & 13000.0 & 260.0 & 155.0 & 12.80 \\
\hline
\end{tabular}

TABLE 3: Distribution of mean white blood cell count and levels of biomarkers in different diagnoses based on clinical diagnosis and paraclinical tests in the nonsurgical group (in patients in which their numbers were $\geq 5$ ).

\begin{tabular}{|c|c|c|c|c|c|}
\hline Final diagnosis & Statistics & WBC Count & Serum S100A8/A9 & Urine S100A8/A9 & Serum amyloid A \\
\hline \multirow{4}{*}{$\begin{array}{l}\text { Complicated ovarian cyst } \\
N=25\end{array}$} & Mean & 9216.0 & 85.28 & 77.0 & 16.88 \\
\hline & Std. deviation & 1640.80 & 68.79 & 61.99 & 17.03 \\
\hline & Minimum & 6000.0 & 19.0 & 8.0 & 4.60 \\
\hline & Maximum & 12000.0 & 282.0 & 229.0 & 78.00 \\
\hline \multirow{4}{*}{$\begin{array}{l}\text { Acute cholecystitis } \\
N=6\end{array}$} & Mean & 12400.0 & 70.0 & 49.33 & 17.53 \\
\hline & Std. deviation & 3384.67 & 61.02 & 41.62 & 11.07 \\
\hline & Minimum & 7400.00 & 29.0 & 13.0 & 7.70 \\
\hline & Maximum & 18000.00 & 189.0 & 125.0 & 39.30 \\
\hline \multirow{4}{*}{$\begin{array}{l}\text { Acute pancreatitis } \\
N=12\end{array}$} & Mean & 13225.00 & 187.42 & 134.42 & 32.95 \\
\hline & Std. deviation & 7097.65 & 142.86 & 45.24 & 35.72 \\
\hline & Minimum & 5500.05 & 50.0 & 48.0 & 7.80 \\
\hline & Maximum & 33800.05 & 559.0 & 238.0 & 129.0 \\
\hline \multirow{4}{*}{$\begin{array}{l}\text { Renal colic } \\
N=6\end{array}$} & Mean & 7000.0 & 60.17 & 47.33 & 8.57 \\
\hline & Std. deviation & 1454.65 & 27.88 & 26.49 & 3.72 \\
\hline & Minimum & 4500.0 & 23.0 & 16.0 & 4.80 \\
\hline & Maximum & 8200.0 & 94.0 & 94.0 & 15.70 \\
\hline \multirow{4}{*}{$\begin{array}{l}\text { Dyspepsia } \\
N=9\end{array}$} & Mean & 10122.22 & 43.45 & 30.44 & 22.98 \\
\hline & Std. deviation & 1856.60 & 26.06 & 20.21 & 22.89 \\
\hline & Minimum & 7800.0 & 23.0 & 9.0 & 4.80 \\
\hline & Maximum & 13300.0 & 91.0 & 77.0 & 71.40 \\
\hline \multirow{4}{*}{$\begin{array}{l}\text { Ileus } \\
N=5\end{array}$} & Mean & 9060.0 & 106.400 & 104.2000 & 21.8400 \\
\hline & Std. deviation & 2822.76 & 69.6046 & 42.67552 & 26.92142 \\
\hline & Minimum & 4800.0 & 50.0 & 51.00 & 7.80 \\
\hline & Maximum & 12000.0 & 208.0 & 154.00 & 69.70 \\
\hline \multirow{4}{*}{$\begin{array}{l}\text { Nonspecific abdominal pain } \\
N=29\end{array}$} & Mean & 8724.14 & 79.83 & 59.35 & 17.15 \\
\hline & Std. deviation & 3032.92 & 64.75 & 59.64 & 17.57 \\
\hline & Minimum & 5200.0 & 12.0 & 9.00 & 6.50 \\
\hline & Maximum & 21000.0 & 314.0 & 213.0 & 93.0 \\
\hline
\end{tabular}


TABLE 4: Distribution of mean white blood cell count and levels of biomarkers in common diagnoses based on the surgical and the nonsurgical groups.

\begin{tabular}{|c|c|c|c|c|c|c|}
\hline Common diagnosis & Group & Statistics & WBC count & Serum S100A8/A9 & Urine S100A8/A9 & Serum amyloid A \\
\hline \multirow{9}{*}{ Complicated ovarian cyst } & \multirow{4}{*}{$\begin{array}{l}\text { Without surgery } \\
\qquad N=25\end{array}$} & Mean & 9216.0 & 85.28 & 77.0 & 16.88 \\
\hline & & Std. deviation & 1640.80 & 68.79 & 61.99 & 17.03 \\
\hline & & Minimum & 6000.0 & 19.0 & 8.0 & 4.60 \\
\hline & & Maximum & 12000.0 & 282.0 & 229.0 & 78.0 \\
\hline & \multirow{5}{*}{$\begin{array}{c}\text { Surgery } \\
N=2\end{array}$} & Mean & 12500.0 & 185.0 & 169.0 & 3.55 \\
\hline & & Std. deviation & 424.26 & 59.40 & 84.85 & 4.46 \\
\hline & & Minimum & 12200.0 & 143.0 & 109.0 & 0.40 \\
\hline & & Maximum & 12800.0 & 227.0 & 229.0 & 6.70 \\
\hline & & $P$-value $¥$ & $0.006^{*}$ & 0.07 & 0.09 & 0.50 \\
\hline \multirow{9}{*}{ Acute cholecystitis } & \multirow{4}{*}{$\begin{array}{l}\text { Without surgery } \\
\qquad N=6\end{array}$} & Mean & 12400.0 & 70.0 & 49.33 & 17.53 \\
\hline & & Std. deviation & 3384.67 & 61.02 & 41.62 & 11.07 \\
\hline & & Minimum & 7400.0 & 29.0 & 13.00 & 7.70 \\
\hline & & Maximum & 18000.0 & 189.0 & 125.00 & 39.30 \\
\hline & \multirow{5}{*}{$\begin{array}{c}\text { Surgery } \\
N=11\end{array}$} & Mean & 19318.12 & 244.91 & 140.73 & 17.75 \\
\hline & & Std. deviation & 24309.09 & 144.94 & 20.11 & 11.99 \\
\hline & & Minimum & 10000.0 & 138.0 & 110.0 & 7.60 \\
\hline & & Maximum & 92500.0 & 545.0 & 169.0 & 40.60 \\
\hline & & $P$ value ${ }^{¥}$ & 0.99 & $0.005^{*}$ & $0.001^{*}$ & 0.88 \\
\hline \multirow{6}{*}{ Ectopic pregnancy } & \multirow{4}{*}{$\begin{array}{l}\text { Without surgery } \\
\qquad N=3\end{array}$} & Mean & 13566.67 & 149.0 & 150.33 & 33.20 \\
\hline & & Std. deviation & 4377.60 & 87.02 & 54.86 & 22.23 \\
\hline & & Minimum & 9500.0 & 63.0 & 111.0 & 9.70 \\
\hline & & Maximum & 18200.0 & 237.0 & 213.0 & 53.90 \\
\hline & \multirow{2}{*}{$\begin{array}{c}\text { Surgery } \\
N=1\end{array}$} & Mean & 16000.0000 & 336.000 & 251.0000 & 7.9000 \\
\hline & & $P$ value ${ }^{¥}$ & 0.990 & 0.50 & 0.50 & 0.50 \\
\hline
\end{tabular}

${ }^{*}$ Statistically significant $P$ value. ${ }^{¥} P$ value from the Mann-Whitney $U$ test.

TABLE 5: Distribution of the mean time of onset of pain until patient referral on the levels of white blood cell count and biomarker between the surgical and the nonsurgical groups.

\begin{tabular}{|c|c|c|c|}
\hline Group & Variable & Correlation $/ P$ value ${ }^{¥}$ & Time \\
\hline \multirow{8}{*}{$\begin{array}{l}\text { Without surgery } \\
N=110\end{array}$} & \multirow{2}{*}{ WBC count } & Correlation coefficient & 0.130 \\
\hline & & $P$ value & 0.175 \\
\hline & \multirow{2}{*}{ Serum S100A8/A9 } & Correlation coefficient & 0.225 \\
\hline & & $P$ value & $0.018^{*}$ \\
\hline & \multirow{2}{*}{ Urine S100A8/A9 } & Correlation coefficient & 0.274 \\
\hline & & $P$ value & $0.004^{*}$ \\
\hline & \multirow{2}{*}{ Serum amyloid A } & Correlation coefficient & 0.270 \\
\hline & & $P$ value & $0.004^{*}$ \\
\hline \multirow{8}{*}{$\begin{array}{l}\text { Surgery } \\
N=71\end{array}$} & \multirow{2}{*}{ WBC count } & Correlation coefficient & -0.028 \\
\hline & & $P$ value & 0.816 \\
\hline & \multirow{2}{*}{ Serum S100A8/A9 } & Correlation coefficient & -0.253 \\
\hline & & $P$ value & $0.034^{*}$ \\
\hline & \multirow{2}{*}{ Urine S100A8/A9 } & Correlation coefficient & -0.193 \\
\hline & & $P$ value & 0.106 \\
\hline & \multirow{2}{*}{ Serum amyloid A } & Correlation coefficient & -0.116 \\
\hline & & $P$ value & 0.335 \\
\hline
\end{tabular}

*Statistically significant $P$ value. ${ }^{¥} P$ value from the Mann-Whitney $U$ test. 
patient's arrival, with the serum and urine levels of S100A8/ A9 and the serum amyloid A biomarkers. In other words, with increased "mean time of onset of pain until patient arrival," the mean levels of serum and urine S100A8/A9 and serum amyloid A biomarkers will increase and vice versa.

However, in the surgical group, there was a significant inverse correlation between the "mean time of pain onset until patient arrival" and the mean levels of the serum S100A8/A9 biomarker.

A strong correlation between the relative increases of S100A8/A9 and that of WBC in PSI+/-, but not in PNSI $+/-$, was found. Interestingly, the correlation of the increase in S100A8/A9 and that of serum amyloid A progressed across the two groups being nonsignificant in group PNSI $+/-$ and moderately to highly significant in group PSI+/(Table 6).

Finally, the results of the ROC curve analysis showed that in anticipation of the need for surgery in acute abdominal pain, the diagnostic accuracy of the WBC count, serum S100A8/A9, urine S100A8/A9, and serum amyloid A were $71 \%, 86 \%, 79 \%$, and $50 \%$, respectively (Figure 2 ).

The summary of diagnostic values of each biomarker in the study participants has been given in Table 7 .

\section{Discussion}

The studied biomarkers are produced and secreted in response to the immune system stimulation and continuation of the inflammatory processes; therefore, the measurement of their presence and quantity in body fluids and tissues can help in the early diagnosis and prognosis of some diseases.

In the present study, in contrast to other studies that considered a specific diagnosis, we considered a wider range of patients with acute abdominal pain in terms of the final outcome and biomarkers. Our results showed that in many other diagnoses in the abdominal and pelvic areas as well as acute appendicitis, which accounts for most patients, the mean levels of the S100A8/A9 biomarker increase. In fact, it is assumed that the diagnoses mentioned are associated with infectious or inflammatory processes and immune system stimulation, which have further led to increased levels of S100A8/A9.

In recent years, S100A8/A9 was studied as a biomarker secreted in serum, urine, and feces in some infectious or inflammatory diseases of the gastrointestinal tract, like inflammatory bowel disease, familial Mediterranean fever, appendicitis, infections, bowel inflammation, and so on, especially in children. Some of these studies confirmed the role of S100A8/A9 in the diagnosis, clinical course, and prognosis of the disease $[7,31,32,37,38]$. Other studies have emphasized the importance of signs and symptoms of the disease and the use of other diagnostic methods and routine biomarkers [32-35].

In this study, we also showed that the levels of serum amyloid A increase in various diagnoses with activated inflammatory or immune responses.

Mayer et al. showed that serum amyloid A can predict the severity of the disease better than CRP, in acute pancreatitis [39]. In addition, Sit et al. showed that although clinical signs
TABLE 6: Spearman rank correlation coefficients of the relative increase of S100A8/A9 to those of WBC and serum amyloid A.

\begin{tabular}{lcccccc}
\hline \multirow{2}{*}{ Variables } & \multicolumn{3}{c}{ PSI + /- } & \multicolumn{3}{c}{ PNSI $+/-$} \\
& $N$ & $r$ & $P$ value & $N$ & $R$ & $P$ value \\
\hline WBC & 52 & 0.901 & 0.002 & 23 & 0.432 & 0.060 \\
Serum amyloid A & 44 & 0.58 & 0.01 & 30 & 0.438 & 0.064 \\
\hline
\end{tabular}

PSI+/- (PSI, pain in the surgical intervention group), comprising subjects in whom acute abdominal pain remitted postsurgically, and PNSI+/- (PNSI, pain in the nonsurgical intervention group), consisting of those subjects who were acute abdominal pain remitted after nonsurgical intervention.

and symptoms play a great role in the diagnosis of acute appendicitis, the measurement of serum amyloid A can help surgeons avoid unnecessary laparotomy [18].

The results of our study showed that the mean levels of serum and urine of S100A8/A9 biomarkers and the WBC count in the surgical group were considerably more than those in the nonsurgical group and can prove to be useful in anticipating the need for surgery in patients with acute abdominal pain, along with other factors. However, serum amyloid A does not seem helpful in this regard.

The heterogeneities in the surgical and the nonsurgical groups, among some diagnoses with inflammatory natures and the levels of biomarkers, may be due to the difference in the time of measurement of the samples, including the onset of the inflammatory process, confounding factors, and technical problems associated with their measurement.

In our study, some diagnoses were common in the surgical and the nonsurgical groups when levels of biomarkers where compared.

In "abdominal pain caused by complicated ovarian cysts," the mean level of serum and urine of S100A8/A9 and the WBC count were higher than normal in the surgical group. But in the nonsurgical group, only the WBC count and serum amyloid A were higher. Finally, it can be stated that regarding the statistical significance, only the WBC count can predict the need for surgery in complicated ovarian cyst.

In the diagnosis of "abdominal pain caused by acute cholecystitis," the mean level of serum and urine of S100A8/A9, the WBC count, and serum amyloid A were higher than normal in the surgical group and only the WBC count and serum amyloid were higher than normal in the nonsurgical group. Statistically, it seems that only the serum and urine levels of the S100A8/A9 biomarker can predict the need for surgery in acute cholecystitis.

In the diagnosis of "abdominal pain caused by ectopic pregnancy," the mean level of serum and urine of S100A8/ A9 and the WBC count were higher than normal in both the surgical and the nonsurgical groups. However, the mean level of serum amyloid A was higher only in the nonsurgical group. Statistically, none of these indicators can be useful in predicting the need for surgery in ectopic pregnancy.

Finally, the results of this study showed that the highest diagnostic accuracy for surgery requirement was related to the serum S100A8/A9 biomarker, followed by the urine S100A8/A9 biomarker. Serum amyloid A biomarker had a low and nonsignificant diagnostic accuracy that may be valuable in combination with other biomarkers. 


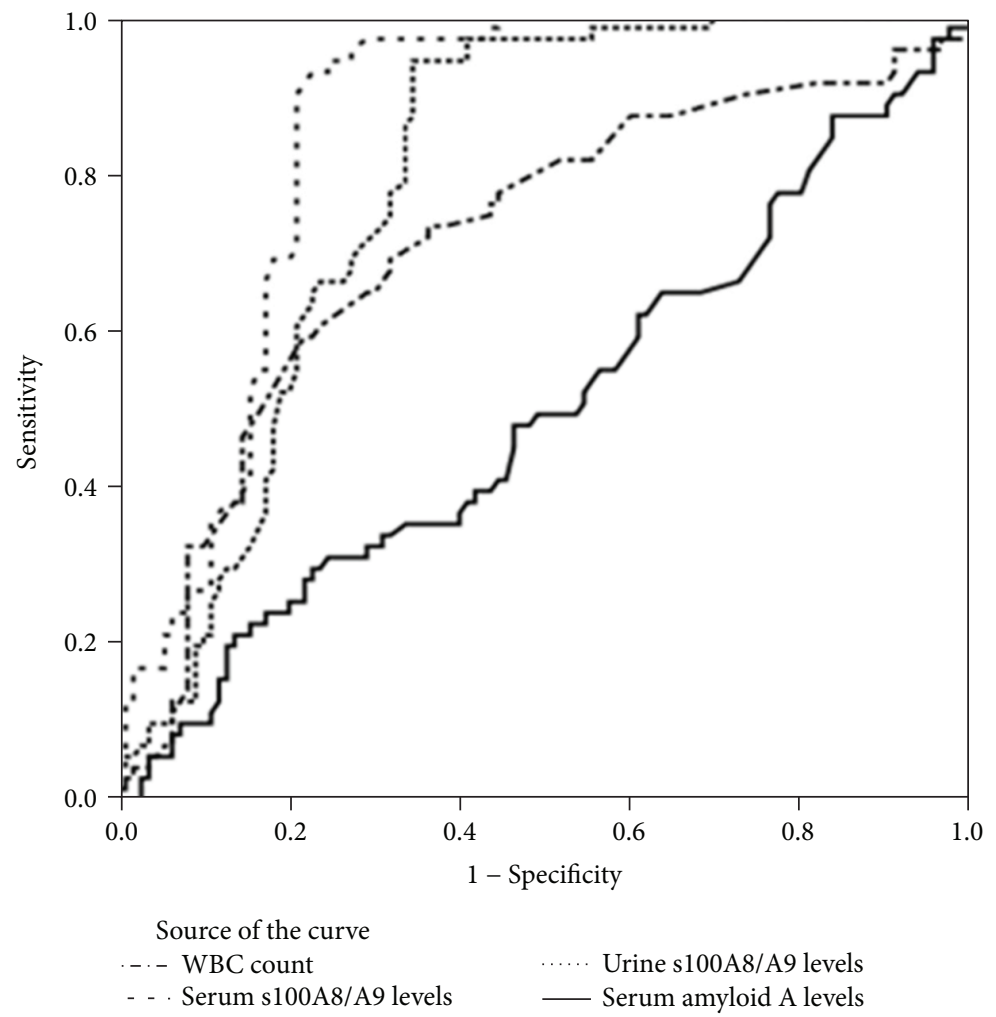

FIGURE 2: Diagnostic accuracy of white blood cells and serum and urinary biomarkers in the diagnosis of acute abdominal pain requiring surgery.

TABLE 7: Summary of diagnostic accuracy of various biomarkers of interest in the study group.

\begin{tabular}{lcccccc}
\hline Test result variable(s) & AUC & SE & Sensitivity & Specificity & $P$ value & $\begin{array}{c}\text { Asymptotic } 95 \% \text { confidence } \\
\text { interval } \\
\text { Lpper bound }\end{array}$ \\
\hline WBC count $\left(\times 10^{9} / \mathrm{L}\right)$ & 0.713 & 0.040 & $97.5 \%$ & $76 \%$ & $<0.001$ & 0.634 \\
Serum S100A8/A9 & 0.855 & 0.028 & $91.5 \%$ & $87.2 \%$ & $<0.001$ & 0.799 \\
Urine S100A8/A9 & 0.791 & 0.033 & $93 \%$ & $77.5 \%$ & $<0.001$ & 0.725 \\
Serum amyloid A & 0.499 & 0.045 & $33.6 \%$ & $43.5 \%$ & .984 & 0.412 \\
\hline
\end{tabular}

The results of our study showed that in the nonsurgical group, the levels of biomarkers had a weak positive association with the mean time of pain onset until the arrival of the patient. In other words, the longer the onset of the patient's pain until arrival, the greater the levels of biomarkers.

But among the patients in the surgical group, the levels of biomarkers had a weak negative association with the onset of the patient's pain until arrival, which means that the longer the onset of the patient's pain until arrival, the lower the levels of biomarkers.

In the nonsurgical group, only the WBC count had a nonsignificant positive association, but in the surgical group, serum S100A8/A9 alone had a significantly inverse relationship (Table 5).

Assessment of the mean time of onset of pain among patients until arrival in both the surgical and the nonsurgical groups and the time of the measurement of the biomarkers resulted in different results that cannot easily be justified.
Our findings showed that S100A8/A9 but not amyloid A is increased in the surgical group. Though we cannot prove a causal relation for these findings, the possible reason is that amyloid $\mathrm{A}$ is an acute-phase reactant that is produced in the liver but, in patients of the surgical group with mentioned specific diagnoses, could not stimulate the liver for enough production. More studies are needed for clarification.

Mills et al. [7] investigated the role of S100A8/A9 biomarkers in patients with acute lower abdominal pain and found that the problems of purchase, transfer of the kits, the shipping effect, and the delay in sample analysis of the biomarkers increase the sensitivity and decrease the specificity and the diagnostic accuracy of the test.

\section{Limitations}

The heterogeneous composition of the two groups with certain diagnoses in both groups and the low number of cases 
limit the validity of our study. Also, in our study, due to the problems associated with the purchase and shipment of the kits, the costs, and the technical limitations of measurement, sampling was performed at the baseline. After reaching the sample size, the levels of biomarkers were simultaneously measured. Logically, any delay in the analysis of the sample should, perhaps, lead to increased levels of biomarkers, due to cell lysis and the release of the cellular contents. Also, it seems pharmacokinetic and pharmacodynamic characteristics of biomarkers and inflammation severity of patients affect the biomarker levels in correlation with the onset of pain times. We could not explain these differences and discrepancies in the levels of biomarkers with respect to the pain onset and admission time.

\section{Conclusion}

Though our data may show that the serum and urine S100A8/A9 can be useful indicators of the need for surgery in patients presenting with acute abdominal pain at the emergency department, the data rather suggest that patients in the surgical group were more seriously ill than those in the nonsurgical group, with a correspondingly higher risk of operation. Serum amyloid A had a low and nonsignificant diagnostic accuracy, which may be valuable in combination with clinical findings and other biomarkers.

\section{Disclosure}

This manuscript was prepared from the postgraduate thesis of Dr. Amirali Fallah Amoli, Dr. Niloofar Akhiani, and Dr. Fatemeh Ghourchian under the supervision of Ahvaz Jundishapur University of Medical Sciences.

\section{Conflicts of Interest}

All authors declare that they have no competing interests.

\section{Acknowledgments}

Special thanks are due to Ahvaz Imam Khomeini Hospital Clinical Research Development Unit (CREDI).

\section{Supplementary Materials}

Supplementary material included two tables. They show the distribution of mean white blood cell count and the levels of biomarkers in different diagnoses based on pathological findings in the surgical and the nonsurgical groups in patients in which their numbers were less than 5 . In the surgical group (Table 1 of Supplementary Material), some diagnoses were less than 5 in number, in which biomarker levels were calculated and recorded for them and used for the final analysis. They included complicated ovarian cyst, volvulus, stomach perforation, ectopic pregnancy, colon tubuadenomatosis tumor, mesenteric ischemia, pancreas abscess, complicated ovarian tumor, and duodenal perforation. Also, in the nonsurgical group (Table 2 of Supplementary Material), some diagnoses were less than 5 in number, in which biomarker levels were calculated and recorded for them and used for the final analysis. They included ectopic pregnancy, acute cholangitis, surgery site abscess, gastritis, acute hepatitis, urinary tract infection, biliary colic, incarcerated periumbilical hernia, varicocele, pelvic inflammatory disease, and mittelschmerz. (Supplementary Materials)

\section{References}

[1] M. John Marx, M. D. Robert Hockberger, and M. D. Ron Walls, Rosen's Emergency Medicine - Concepts and Clinical Practice, Elsevier Publishing, 8th edition, 2014.

[2] M. Kulla, S. Kraus, F. Walcher et al., "Patients with acute, nontraumatic abdominal pain in German emergency departments: a prospective monocentric observation study," Zentralblatt für Chirurgie, vol. 141, no. 6, pp. 666-676, 2016.

[3] P. C. Ambe, D. Godde, L. Bonicke, M. Papadakis, S. Storkel, and H. Zirngibl, "S100A8/A9 could be a potential biomarker for acute appendicitis," Journal of Translational Medicine, vol. 107, no. 1, p. 141, 2016.

[4] S. L. Gans, M. A. Pols, J. Stoker, M. A. Boermeester, and on behalf of the expert steering group, "Guideline for the diagnostic pathway in patients with acute abdominal pain," Digestive Surgery, vol. 32, no. 1, pp. 23-31, 2015.

[5] G. Cervellin, R. Mora, A. Ticinesi et al., "Epidemiology and outcomes of acute abdominal pain in a large urban emergency department: retrospective analysis of 5,340 cases," Annals of Translational Medicine, vol. 4, no. 19, p. 362, 2016.

[6] N. Caporale, A. M. Morselli-Labate, E. Nardi, R. Cogliandro, M. Cavazza, and V. Stanghellini, "Acute abdominal pain in the emergency department of a university hospital in Italy," United European Gastroenterology Journal, vol. 4, no. 2, pp. 297-304, 2016.

[7] A. M. Mills, D. S. Huckins, H. Kwok et al., "Diagnostic characteristics of S100A8/A9 in a multicenter study of patients with acute right lower quadrant abdominal pain," Academic Emergency Medicine, vol. 19, no. 1, pp. 48-55, 2012.

[8] C. Falch, D. Vicente, H. Häberle et al., “Treatment of acute abdominal pain in the emergency room: a systematic review of the literature," European Journal of Pain, vol. 18, no. 7, pp. 902-913, 2014.

[9] J. S. Kim, "Acute abdominal pain in children," Pediatric Gastroenterology, Hepatology \& Nutrition, vol. 16, no. 4, pp. 219-224, 2013.

[10] A. Bhangu, K. Soreide, S. Di Saverio, J. H. Assarsson, and F. T. Drake, "Acute appendicitis: modern understanding of pathogenesis, diagnosis, and management," The Lancet, vol. 386, no. 10000, pp. 1278-1287, 2015.

[11] J. Benito, Y. Acedo, L. Medrano, E. Barcena, R. P. Garay, and E. A. Arri, "Usefulness of new and traditional serum biomarkers in children with suspected appendicitis," The American Journal of Emergency Medicine, vol. 34, no. 5, pp. 871-876, 2016.

[12] M. Salo, B. Roth, P. Stenstrom, E. Arnbjornsson, and B. Ohlsson, "Urinary biomarkers in pediatric appendicitis," Pediatric Surgery International, vol. 32, no. 8, pp. 795-804, 2016.

[13] O. R. Herrera, M. L. Christensen, and R. A. Helms, "Calprotectin: clinical applications in pediatrics," The Journal of Pediatric Pharmacology and Therapeutics, vol. 21, no. 4, pp. 308-321, 2016. 
[14] D. H. S. M. Schellekens, K. W. E. Hulsewé, B. A. C. van Acker et al., "Evaluation of the diagnostic accuracy of plasma markers for early diagnosis in patients suspected for acute appendicitis," Academic Emergency Medicine, vol. 20, no. 7, pp. 703-710, 2013.

[15] A. B. Kharbanda, A. J. Rai, Y. Cosme, K. Liu, and P. S. Dayan, "Novel serum and urine markers for pediatric appendicitis," Academic Emergency Medicine, vol. 19, no. 1, pp. 56-62, 2012.

[16] G. Thuijls, J. P. M. Derikx, F. J. Prakken et al., “A pilot study on potential new plasma markers for diagnosis of acute appendicitis," The American Journal of Emergency Medicine, vol. 29, no. 3, pp. 256-260, 2011.

[17] H. H. Nordal, K. A. Brokstad, M. Solheim, A. K. Halse, T. K. Kvien, and H. B. Hammer, "Calprotectin (S100A8/A9) has the strongest association with ultrasound-detected synovitis and predicts response to biologic treatment: results from a longitudinal study of patients with established rheumatoid arthritis," Arthritis Research \& Therapy, vol. 19, no. 1, p. 3, 2017.

[18] M. Sit, O. Catal, G. Aktas, E. E. Yilmaz, M. Tosun, and H. Savli, "Serum amyloid A and omentin levels in acute appendicitis: a preliminary study for a novel diagnostic approach," La Clinica Terapeutica, vol. 165, no. 1, pp. e35-e38, 2014.

[19] M. H. Abbas, M. N. Choudhry, N. Hamza, B. Ali, A. A. Amin, and B. J. Ammori, "Admission levels of serum amyloid A and procalcitonin are more predictive of the diagnosis of acute appendicitis compared with C-reactive protein," Surgical Laparoscopy, Endoscopy \& Percutaneous Techniques, vol. 24, no. 6, pp. 1-94, 2014.

[20] F. S. Lehmann, E. Burri, and C. Beglinger, "The role and utility of faecal markers in inflammatory bowel disease," Therapeutic Advances in Gastroenterology, vol. 8, no. 1, pp. 23-36, 2014.

[21] I. Striz and I. Trebichavsky, "Calprotectin - a pleiotropic molecule in acute and chronic inflammation," Physiological Research, vol. 53, no. 3, pp. 245-253, 2004.

[22] W. Marshall, M. Lapsley, A. Day, and R. Ayling, Clinical Biochemistry: Metabolic and Clinical Aspects, Elsevier Health Sciences, 3rd edition, 2014.

[23] R. Gupta, Biomarkers in Toxicology, Academic Press, San Diego, CA, USA, 2014.

[24] G. O. Evans, Animal Clinical Chemistry: A Practical Handbook for Toxicologists and Biomedical Researchers, Taylor \& Francis, Boca Raton, 2nd edition, 2009.

[25] M. B. Brophy and E. M. Nolan, "Manganese and microbial pathogenesis: sequestration by the mammalian immune system and utilization by microorganisms," ACS Chemical Biology, vol. 10, no. 3, pp. 641-651, 2015.

[26] M. R. Elio, T. Pauls, and B. Schwaller, Guidebook to the Calcium-Binding Proteins, Sambrook \& Tooze Publication at Oxford University Press, Oxford, 1996.

[27] C. M. Uhlar and A. S. Whitehead, "Serum amyloid A, the major vertebrate acute-phase reactant," European Journal of Biochemistry, vol. 265, no. 2, pp. 501-523, 1999.

[28] A. K. Dettmar, E. Binder, F. R. Greiner et al., "Protection of human podocytes from Shiga toxin 2-induced phosphorylation of mitogen-activated protein kinases and apoptosis by human serum amyloid P component," Infection and Immunity, vol. 82, no. 5, pp. 1872-1879, 2014

[29] B. Omer, S. Genc, O. Takmaz et al., "The diagnostic role of human epididymis protein 4 and serum amyloid-A in earlystage endometrial cancer patients," Tumour Biology, vol. 34, no. 5 , pp. 2645-2650, 2013.
[30] J. F. Bealer and M. Colgin, "S100A8/A9: a potential new diagnostic aid for acute appendicitis," Academic Emergency Medicine, vol. 17, no. 3, pp. 333-336, 2010.

[31] D. S. Huckins, H. K. Simon, K. Copeland, D. M. Spiro, J. Gogain, and M. Wandell, "A novel biomarker panel to rule out acute appendicitis in pediatric patients with abdominal pain," The American Journal of Emergency Medicine, vol. 31, no. 9, pp. 1368-1375, 2013.

[32] D. Horner and A. M. Long, "Towards evidence-based emergency medicine: best BETs from the Manchester Royal Infirmary.BET 3: super calprotectin will not expedite your discharge," Emergency Medicine Journal, vol. 30, no. 8, pp. 691-693, 2013.

[33] E. Olafsdottir, L. Aksnes, G. Fluge, and A. Berstad, "Faecal calprotectin levels in infants with infantile colic, healthy infants, children with inflammatory bowel disease, children with recurrent abdominal pain and healthy children," Acta Paediatrica, vol. 91, no. 1, pp. 45-50, 2002.

[34] B. Makay, O. Makay, and E. Unsal, "Can we use faecal calprotectin to distinguish abdominal pain of familial Mediterranean fever (FMF) from acute appendicitis?," Clinical Rheumatology, vol. 28, no. 2, pp. 239-240, 2009.

[35] G. Meucci, R. D’Incà, R. Maieron et al., "Diagnostic value of faecal calprotectin in unselected outpatients referred for colonoscopy: a multicenter prospective study," Digestive and Liver Disease, vol. 42, no. 3, pp. 191-195, 2010.

[36] M. Manz, E. Burri, C. Rothen et al., "Value of fecal calprotectin in the evaluation of patients with abdominal discomfort: an observational study," BMC Gastroenterology, vol. 12, no. 1, p. 5, 2012.

[37] P. C. Ambe, D. Gödde, L. Bönicke, M. Papadakis, S. Störkel, and H. Zirngibl, "Calprotectin could be a potential biomarker for acute appendicitis," Journal of Translational Medicine, vol. 14, no. 1, p. 107, 2016.

[38] R. K. McCann, K. Smith, and D. R. Gaya, "A prospective single centre pilot evaluation of a serum calprotectin assay in unselected GI patients," Clinical Biochemistry, vol. 50, no. 9, pp. 533-536, 2017.

[39] J. M. Mayer, M. Raraty, J. Slavin et al., "Serum amyloid A is a better early predictor of severity than C-reactive protein in acute pancreatitis," The British Journal of Surgery, vol. 89, no. 2, pp. 163-171, 2002. 


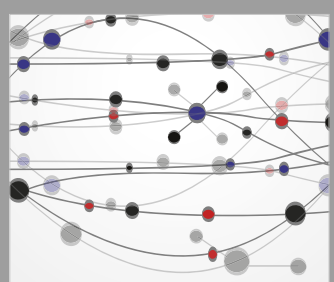

The Scientific World Journal
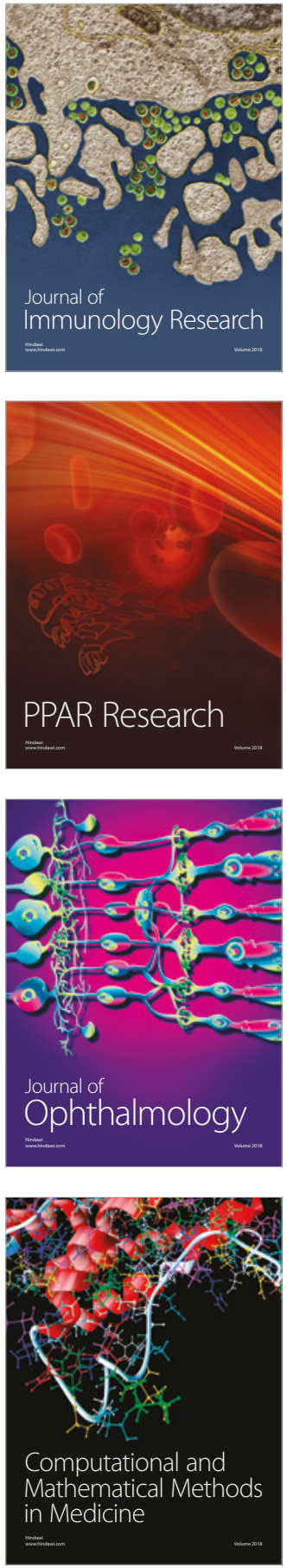

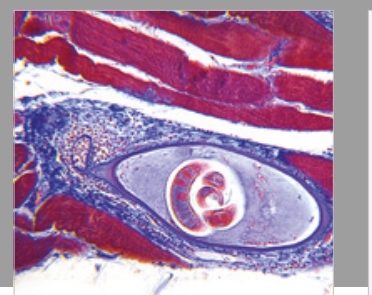

Gastroenterology Research and Practice

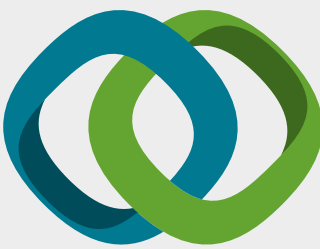

\section{Hindawi}

Submit your manuscripts at

www.hindawi.com
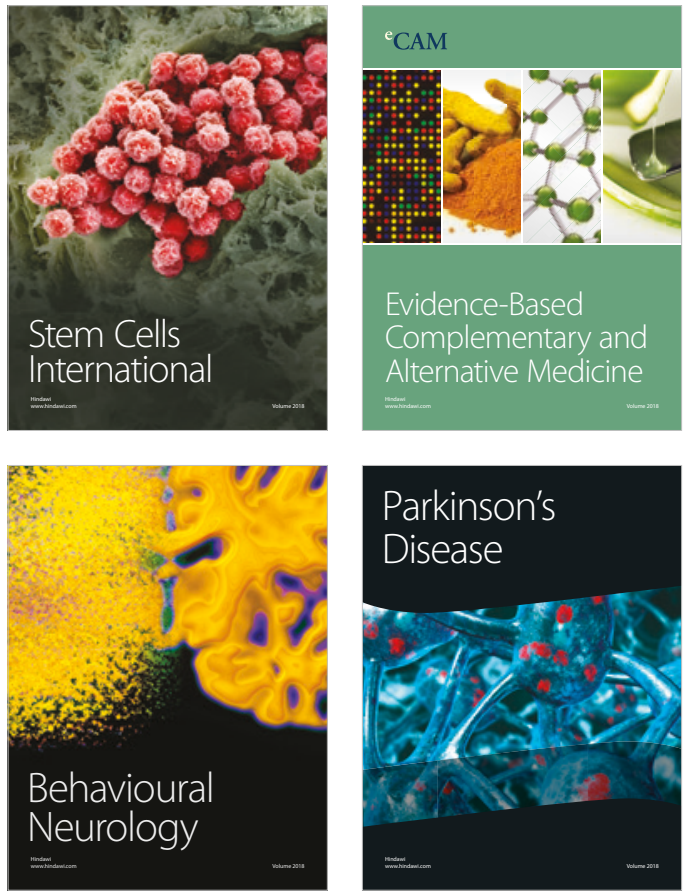

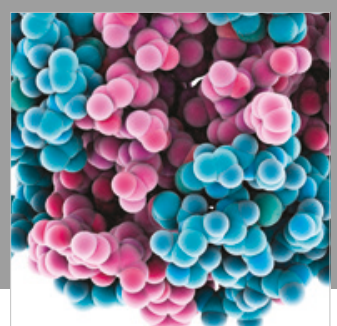

ournal of

Diabetes Research

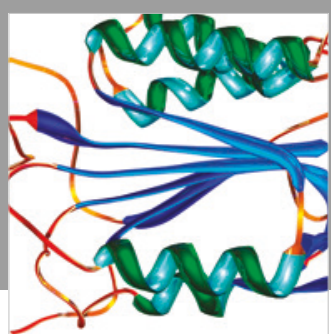

Disease Markers
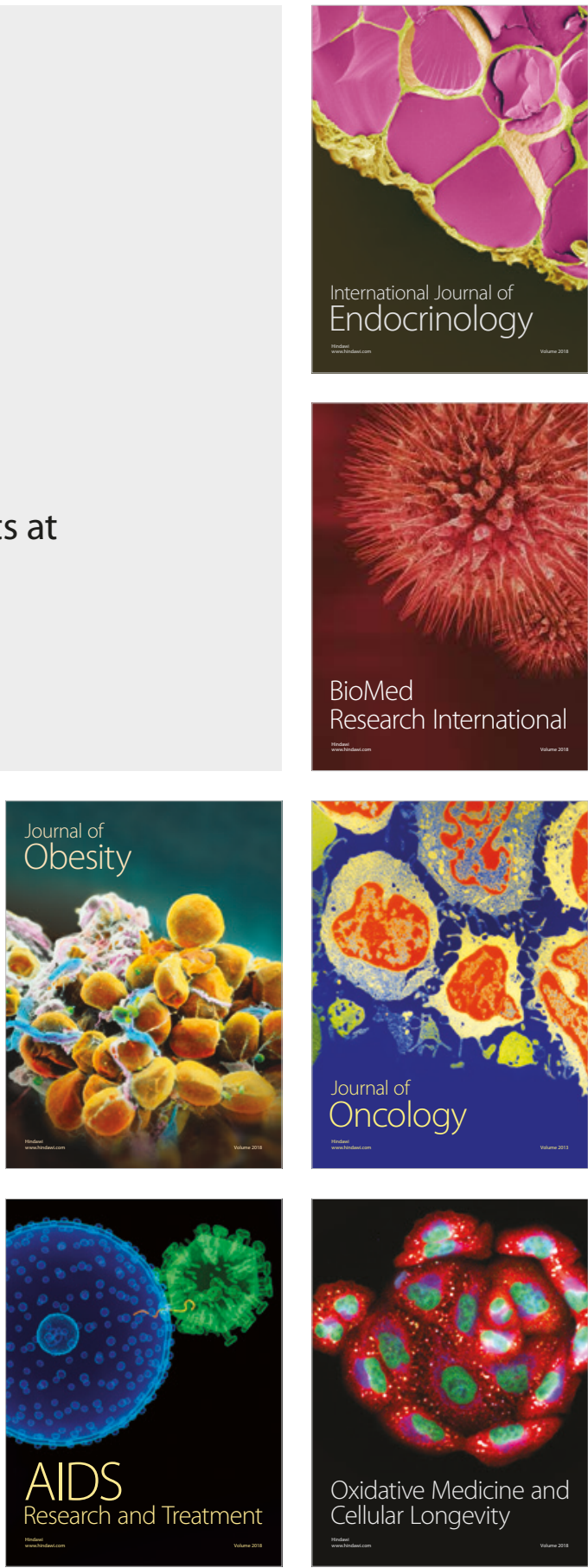